\title{
The effect of meal timing on postprandial glucose metabolism in healthy volunteers
}

\author{
G.K.W. Leung, C.E. Huggins, K.Y. Kong and M.P. Bonham \\ Department of Nutrition and Dietetics, Monash University, Melbourne, Australia
}

Shift workers are at increased risk of chronic conditions including obesity, cardiovascular diseases and type 2 diabetes ${ }^{(1)}$. Compared with a meal consumed during day hours, a meal consumed during the night triggers an exaggerated postprandial glucose response and reduced sensitivity to insulin ${ }^{(2-3)}$. There is evidence to suggest that small changes in the type of food consumed at night can positively impact these responses ${ }^{(4)}$.

The aim of this study, therefore, was to investigate the postprandial glucose response to i) an oral glucose tolerance test (OGTT) and ii) a low glycaemic index (GI) 'healthy' meal challenge, in the morning $(8 \mathrm{am})$ compared to night time $(8 \mathrm{pm})$ in healthy volunteers.

Participants initially completed the OGTT challenge $(n=10)$ and then were invited back to complete the low GI challenge $(n=7)$. Each test-meal challenge was a cross over trial to compare morning and night time responses. Prior to each test-meal challenge, a standard meal was provided and then participants fasted for 10 hours. Finger prick (OGTT) and intravenous blood samples (low GI meal) for glucose were collected at 7 or 9 time points, respectively (see Figs 1A and 1B). To compare the glucose response between morning and night, the incremental AUC (iAUC) was calculated using the trapezoidal rule and significance tested using Wilcoxon-signed rank. A p -value $<0.05$ was taken as significant.
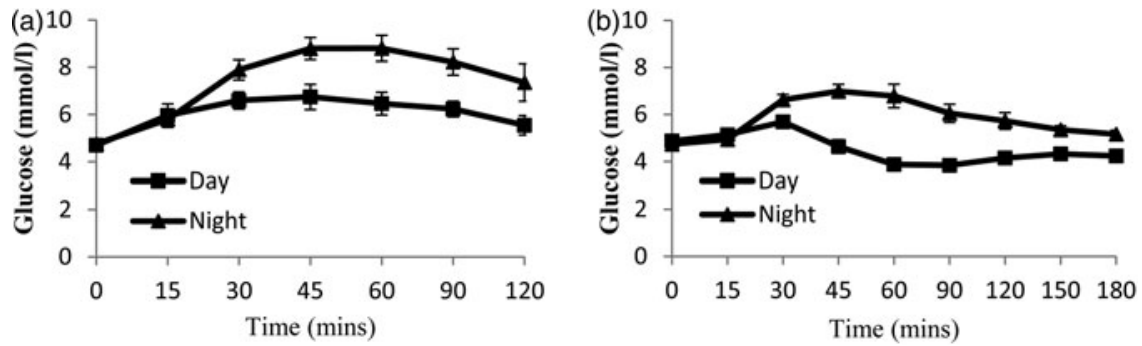

Fig 1: Change in glucose after A) OGTT $(n=10: 8$ F,2M), and B)low GI meal $(n=7: 5$ F,2M). Data $=$ mean \pm SEM

Participants (median, IQR) in the two trials (OGTT (8 females, 2 males) and low GI ( 5 female, 2 male)) were normal weight (BMI $22.6(3.0)$ and $22.9(3.7))$ with a median age of 23 (4) yr and $23.5(5)$ yr respectively.

No differences were observed between fasting glucose at $8 \mathrm{am}$ and $8 \mathrm{pm}$ for both the OGTT and meal study $(\mathrm{p}=0.475$ and $\mathrm{p}=0.175$ respectively). As expected a greater iAUC (median (IQR)) for glucose was observed at night time compared to the morning for the OGTT (332 (166) vs. $181(160) \mathrm{mmol} / 2 \mathrm{~h} \mathrm{p}=0.007))$. Furthermore, the low GI meal at night resulted in a significant increase in iAUC (170 (200) vs. $9(18) \mathrm{mmol} / 3 \mathrm{~h}) \mathrm{p}=0.018)$ ).

These data show that both an oral glucose load and a low GI meal result in exaggerated glucose responses at 8pm compared with $8 \mathrm{am}$. These findings confirm circadian differences in the response to eating but more importantly warrant further research into identifying the optimal timing of both meal intake and meal composition for shift workers.

1. Vyas MV, Garg AX, Iansavichus AV et al. (2012) $B M J$, 345: e4800.

2. Lund J, Arendt J, Hampton SM et al. (2001) J Endocrinol, 171: 557-564.

3. Al-Naimi S, Hampton SM, Richard P et al. (2004) Chronobiol Int 2004, 21: 937-947.

4. Farshchi HR, Taylor MA, Macdonald IA (2005) Am J Clin Nutr 81:16-24. 